\title{
Discourse Analysis in Tamil Speaking Individuals with Parkinson's Disease
}

\author{
Maria J', Samyuktha $\mathbf{N}^{* *}$, Yuva Yoga Shree B***, V. Thiruvalluvan ${ }^{* * * * *}$, \\ Aravind Kannan*: \\ *Assistant Professor, ${ }^{* *}$ Lecturer, ${ }^{* * *}$ Post Graduate Student, ${ }^{* * * * *}$ Professor, MERF-ISH, Chennai. \\ ${ }^{* * * *}$ Professor, CAS in Linguistics, Annamalai University, Chidambaram
}

Corresponding Author: Maria J

\begin{abstract}
Background: Parkinson's disease (PD) is a degenerative disorder of the central nervous system. Though PD is primarily a motor speech disorder during the initial stages, as the disease progresses, a gradual decline in linguistic aspects is observed as well. The effects of disease (PD) on cognition, word retrieval, syntax, and speech/voice processes may act together to manifest uniquely in spoken language tasks. There is a dearth of studies focusing on the discourse abilities in PD, especially in Indian context.
\end{abstract}

Aim: The present study aims at investigating the micro and macrostructure discourse by using personal narrative task in Tamil speaking individuals with idiopathic PD and comparing it with the neuro-typical individuals

Method: Two groups comprising 5 Tamilspeaking individuals with idiopathic PD in the age range of 60-85 years and 5 neuro-typical individuals were included in the study. To assess their discourse skills, participants were engaged in a personal narrative task. The analyses done were based on macro and micro structural aspects of discourse. The raw scores were subjected to suitable statistical analysis.

Results: There was a statistical significance between the Parkinson \& Normal group on Total number of words, No. of Different words, No. of content words, No. of Functional words, Free morphemes, Complex structure per utterances \& Relevant piece per utterance in microstructural aspects. However there was no significant difference observed in the macrostructural aspects due to smaller sample size.
Conclusion: It can be concluded that in addition to speech impairment, language structure is also affected in persons with PD. Intervening at the level of discourse is an important aspect as it enhances the quality of life.

Key words: Parkinson's disease, Discourse, Tamil speaking, Micro analysis, Macro Analysis

\section{INTRODUCTION}

Parkinson's disease (PD) is a neurodegenerative disorder due to disturbance in the dopamine regulation and dysfunction of the basal ganglia system. The disease affects both motor (e.g., movement, walking, speech) and non-motor (e.g., cognition, mood) functions with an obvious effect on health-related quality of life. Dr. James Parkinson, who first defined Parkinson disease (PD) in his seminal paper originally published in the year 1817, fell short of capturing fully the non-motor consequences of the disease particularly those related to cognition and to language, regardless of his detailed explanations of the motor symptoms.

The hallmarks of PD are dopaminergic dysregulation and basal ganglia (BG) dysfunction, PD also affects other neurotransmitter systems having an effect mood regulation and frontal lobe cognitive functions (Zgaljardic, Foldi, \& Borod, 2004). The cognitive processes such as working memory, attention allocation, selection processes, inhibition processes, error detection, error resolution, rule-based 
learning, and sequencing which are associated with language functions have been attributed to the dopamine-mediated Basal ganglion circuits and the prefrontal cortex. Whether or not the BG has specific roles in language processing and production independent of cognitive processes remains a key question.

Angela Roberts et al (2018) reported that the cognitive, language, and motor speech impairments reported in the PD literature underscore the importance of studying the impact of these deficits on discourse performance. Spoken discourse production tasks offer a unique opportunity to advance our understanding of how discrete impairments of cognition, language and motor speech are manifest in spontaneous spoken language tasks. The study conducted by the authors compared the information content and information efficiency of spoken language in individuals with PD to a healthy control group. Results of the study exposed Individuals with PD without dementia showed reduced discourse informativeness that reveals disruptions in both conceptual and lexical discourse processes. In exploratory analyses, reduced efficiency of information content was related with global cognition and motor severity.

Isabelle Caspari, Stanley R. Parkinson in 2000 studied the effects of memory impairments on discourse. The discourse genres included were procedural, narrative and conversation. Since memory plays an important role in the production of a complex behaviour called discourse, they were analysed in words or sentence level (micro linguistics) under which lexical and semantic productivity and syntactic complexity were measured and within texts analysis (macro linguistics) under which cohesion, coherence, side structure analysis, pragmatic analysis and mazing were measured. The results revealed that the patient with amnesia demonstrated some striking differences in discourse production. Although structurally adequate, the discourse was observed to be unelaborated and impoverished. It was concluded that the memory plays an important role in the expression of complex language such as discourse.

In a study done by Vanhoutte et al., (2012), the Parkinson disease patients had a significant difference in the production of semantic perseverations and had low type token ratio which was attributed to the cognitive dysfunction happening in those patients; whereas they did not document significant difference in parameters such as mean length of utterance (MLU), proportion of verbs, Type Token Ratio (TTR) for notional verbs, proportion of nouns, TTR for nouns, proportion of grammatically correct sentences, proportion of content words, and proportion of complex sentences. They also specified that they could observe the production of semantic perseverations and the low type token ratio more significantly in individuals with advance stage of PD which was attributed to cognitive dysfunction and dopamine depletion. Charles Ellis et al, 2015 observed cohesive inadequacy as individuals with PD produced a higher percentage of incomplete and erroneous cohesive ties relative the control subjects across narratives.

Angela Roberts et al (2018) analyzed information content and information efficiency in persons with Parkinson's disease. They were given 2 standardized picture stimuli and asked them narrate a picture from it. The characteristics analyzed include total words, number of Cunits, number of CIU units, total main events, speaking time and total verbs. It was revealed that the persons with PD without dementia demonstrated reduced discourse informativeness that reveals disruptions to both conceptual and lexical discourse processes.

The results of the study conducted by Amulya et al in 2017, revealed that there was a significant difference between individuals with Parkinson's disease and neuro-typical individuals on several aspects of the macro and microstructure measures. Specifically there were significant 
difference on Aiming at resolving problems, Proportion of content words, Proportion of functional words, Number of different words and Type token ratio.

The literature review reveals that there are sparse studies focusing on analyzing the language aspects in detail in individuals with Neuro-degenerative conditions. And there are limited researchers done in Indian languages especially in Tamil speaking population.

Therefore the aim of the present study was to document the macrostructural and microstructural aspects of discourse production in Tamil speaking individuals with Parkinson's disease and compare their performance with neuro-typical individuals.

\section{METHOD}

Participants: Two groups comprising 5 Tamil-speaking individuals with idiopathic
Parkinson's Disease diagnosed by a movement disorder neurologist in the age range of 35 to 75 years and 5 neuro-typical individuals matched on age, gender, socioeconomic status and language were included in the study. Individuals with PD were ranging from moderate to severe hypokinetic dysarthria on Frenchay's Dysarthria assessment and their post onset duration ranged from 1 to 10 years. Individuals with other neurological injury, surgical procedure, or any other acquired neurological diseases were excluded from the study. Informed consent were taken from the participants or the caregivers prior to the data collection. The subject characteristics of the individuals with Parkinson's disease included in the study are included in table 1

Table 1: Subjects characteristics of individuals with Parkinson's disease
\begin{tabular}{|c|l|l|l|c|l|c|}
\hline Subject & Age / Sex & Onset & SES & MMSE & Severity of dysarthria & Sensory issue \\
\hline S1 & $60 \mathrm{y} / \mathrm{F}$ & $4 \mathrm{yrs}$ & Upper middle & 25 & Moderate & Nil \\
\hline S2 & $77 \mathrm{y} / \mathrm{f}$ & $1 \mathrm{yr}$ & Upper middle & 9 & Severe & Nil \\
\hline S3 & $74 \mathrm{y} / \mathrm{F}$ & $10 \mathrm{yrs}$ & Upper middle & 19 & Moderate & Nil \\
\hline S4 & $73 \mathrm{y} / \mathrm{F}$ & $10 \mathrm{Yrs}$ & Upper middle & 10 & Severe & Nil \\
\hline S5 & $38 \mathrm{y} / \mathrm{M}$ & $1 \mathrm{Yr}$ & Upper middle & 18 & Moderate & Nil \\
\hline
\end{tabular}

Stimuli: To assess their discourse skills, participants were engaged in a monologue discourse task of personal narrative about 'An unforgettable incident in their life'. The subjects were instructed to at least talk about the topic for 1 minute. The samples were audio recorded.

Transcription and Analysis: All the samples were transcribed orthographically and divided into communication units. The communication units were analysed in terms of macro and micro structural elements of discourse. The macrostructural elements included discourse structure, global coherence, local coherence, topic management, information adequacy, repair strategy, and the microstructural elements included in the study are total number of words, number of different words, number of content words, number of functional words, number of nonsense words, number of bound morphemes, number of free morphemes, complex structure per utterance, relevant pieces per utterance, number of mental state words, number of pronouns, number of ambiguous pronouns and cohesion. The raw scores were documented in excel sheets and were subjected to Mann-Whitney $\mathbf{U}$ test for understanding the statistical significance between control group and the experimental group.

\section{RESULTS}

The results for the present study are discussed under macro and micro structural aspects.

\section{Macrostructure}

The macrostructural elements analysed in the present study were discourse structure, global coherence, local coherence, topic management, information adequacy, and repair strategies used by the individuals.

Though there was a difference observed in the mean raw score were the neuro-typical individuals out performed 
individuals with Parkinson's disease, there were no statistical significance observed between the groups. This can be due to the sample size being less. The mean score for the macrostructure is depicted in Table 2.

Table 2: Mean and SD of macrostructural scores of Neuro-typical individuals and Parkinson's Disease

\begin{tabular}{|l|c|c|c|c|c|}
\hline \multirow{2}{*}{ Domain } & \multicolumn{2}{c|}{ Neuro-Typical Individuals } & \multicolumn{2}{c|}{ Individuals with PD } & \multirow{2}{*}{ P value } \\
\cline { 2 - 5 } & Mean & SD & Mean & SD & \\
\hline Discourse Structure & 5.00 & 0.00 & 3.60 & 1.67 & 0.054 \\
\hline Global Coherence & 5.00 & 0.00 & 3.80 & 1.30 & 0.054 \\
\hline Local Coherence & 5.00 & 0.00 & 3.80 & 1.30 & 0.054 \\
\hline Topic Management & 4.80 & 0.45 & 3.60 & 1.14 & 0.055 \\
\hline Information Adequacy & 5.00 & 0.00 & 3.80 & 1.79 & 0.136 \\
\hline Repair Strategy & 5.00 & 0.00 & 3.60 & 1.67 & 0.054 \\
\hline
\end{tabular}

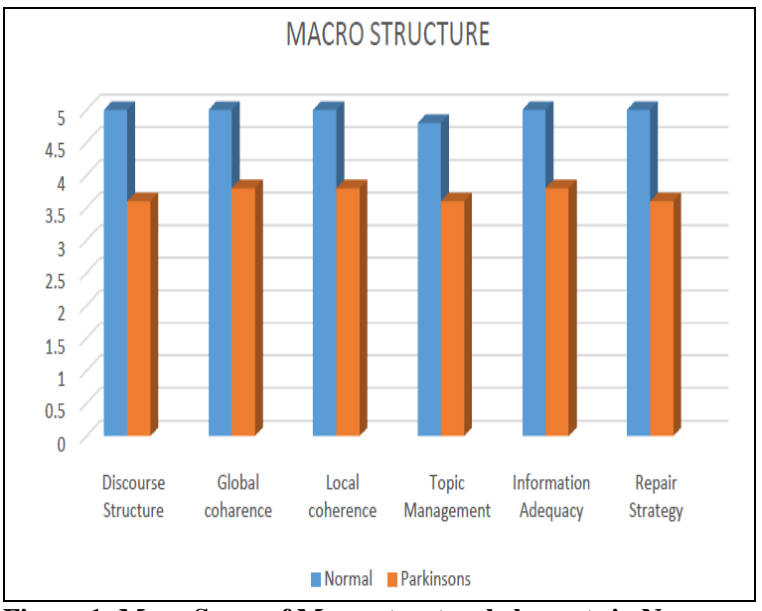

Figure 1: Mean Score of Macrostructural elements in Neurotypical Individuals and Individuals with Parkinson's Disease

\section{Microstructure}

The microstructural elements analysed in the present study are total number of words, number of different words, number of content words, number of functional words, number of nonsense words, number of bound morphemes, number of free morphemes, complex structure per utterance, relevant pieces per utterance, number of mental state words, number of pronouns, number of ambiguous pronouns and cohesion.

Table 3: Mean and SD of microstructural scores of Neuro-typical individuals and Parkinson's disease

\begin{tabular}{|l|c|c|c|c|c|}
\hline \multirow{2}{*}{ Domain } & Neuro-Typical Individuals & \multicolumn{2}{c|}{ Individuals with PD } & \multirow{2}{*}{ P value } \\
\cline { 2 - 5 } & Mean & SD & Mean & SD & \\
\hline Total No. of words & 88.40 & 9.94 & 37.80 & 10.16 & $\mathbf{0 . 0 0 9}$ \\
\hline No. of different words & 64.80 & 10.33 & 30.20 & 3.11 & $\mathbf{0 . 0 0 9}$ \\
\hline No. of content words & 50.00 & 9.82 & 17.20 & 3.96 & $\mathbf{0 . 0 0 9}$ \\
\hline No. of functional words & 18.00 & 6.60 & 10.40 & 2.30 & $\mathbf{0 . 0 3 6}$ \\
\hline No. of Nonsense words & 0.00 & 0.00 & 1.60 & 2.19 & 0.134 \\
\hline No. of Bound morphemes & 19.80 & 13.61 & 10.00 & 4.64 & 0.075 \\
\hline No. of Free morphemes & 100.00 & 7.18 & 35.40 & 12.93 & $\mathbf{0 . 0 0 9}$ \\
\hline Complex structure / Utterance & 3.80 & 0.84 & 0.80 & 0.84 & $\mathbf{0 . 0 0 8}$ \\
\hline Relevant pieces / Utterance & 15.20 & 2.59 & 5.60 & 0.55 & $\mathbf{0 . 0 0 8}$ \\
\hline No. of mental state words & 1.40 & 0.55 & 1.80 & 1.64 & 0.911 \\
\hline No. of Pronouns & 10.20 & 3.03 & 5.00 & 3.54 & 0.059 \\
\hline No. of ambiguous pronouns & 1.20 & 0.84 & 1.40 & 2.19 & 0.661 \\
\hline Cohesion & 0.88 & 0.10 & 0.62 & 0.42 & 0.458 \\
\hline
\end{tabular}

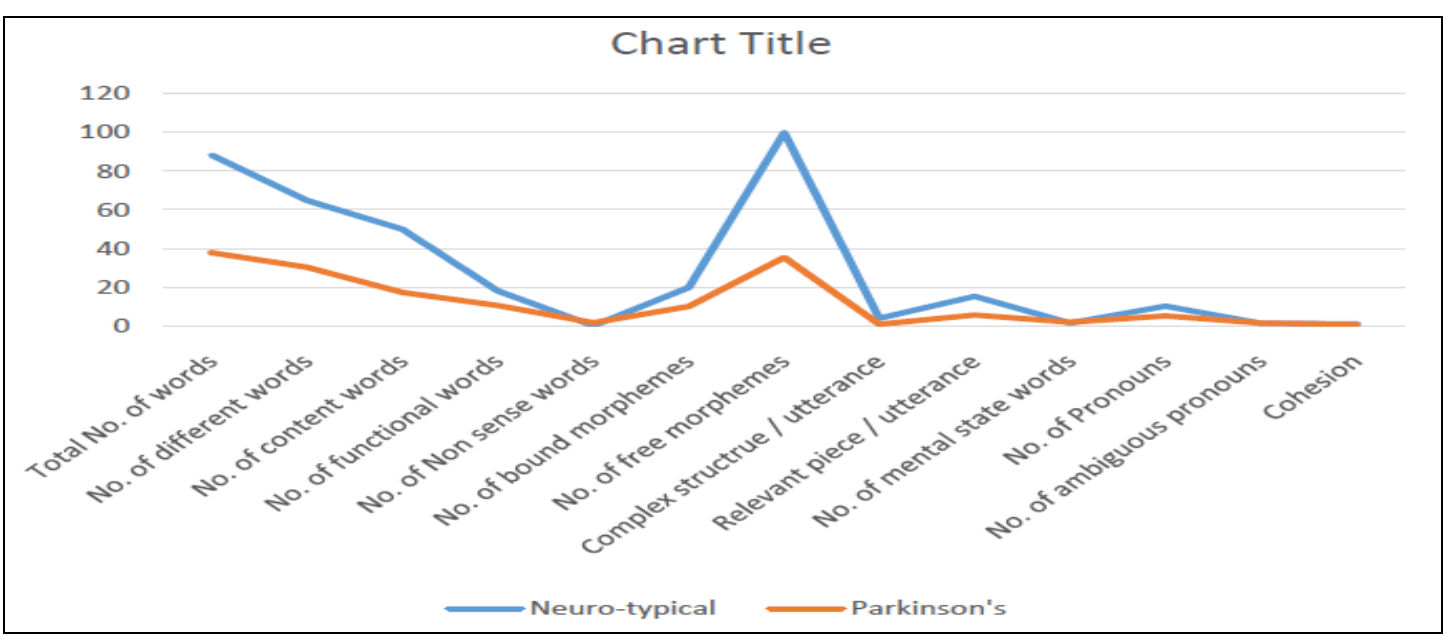

Figure 2: Mean Score of Microstructural elements in Neuro-typical Individuals and Individuals with Parkinson's Disease 
The calculated test statistic value is greater than the critical value that there is a significant difference between the Parkinson \& Normal group on Total number of words (0.009), No. of Different words (0.009), No. of content words (0.009), No. of Functional words (0.036), No. of Free morphemes (0.009), Complex structure per utterances $(0.008)$ and Relevant piece per utterance (0.008).

\section{DISCUSSION}

Changes in cognition and language, occurring in the context of Parkinson disease, affect spontaneous language in a predictable manner that can be uniquely characterized using spoken monologic discourse tasks. The findings of the current study, help us understand more about the communication of individuals with Parkinson's disease, amidst the studies which usually focuses on the motor speech production/ voice parameters.

The current study found a significant difference in the total number of new words, number of different words, number of content words and number of function words; where neuro-typical individuals outperformed individuals with the Parkinson's disease.

Similar study was conducted by Amulya et al (2017) in Kannada a south Indian language which measured the macrolinguistic and microlinguistic abilities in person's with idiopathic Parkinson's disease and found a significant difference in the Microlinguistic aspects such Proportion of content words, Proportion of function words, Total number of different words, Type token ration. The study also revealed that individuals with Parkinson's disease performed better than neuro typical individuals in the use of content words which is in contrast with the current study. This could possibly be attributed to the structured stimuli which were used in the study to elicit discourse production in the previous study. Whereas, the current study had a free recall stimuli, where the participants can freely use words and there was comparatively more cognitive demand. There was a contrasting finding by Murray (2000) who found no significant difference in the total number of utterance by neuro typical individuals and individuals with Parkinson's disease.

Rao, Reddy, and Narayanan (2016) reported that the number of different words to be restricted in individuals with Parkinson's disease; similar finding was observed in the current study, where individuals with PD produced relatively limited number of different words due to lexical retrieval difficulty. The findings of the current study on morpheme production are consistent with the previous findings where persons with Parkinson's disease had difficulty in producing morphemes.

Ash et al., (2017) reported that Parkinsonism with dementia differed from the healthy group in the information content which declined over a course of 36 months, which indicates that individuals with PD produced simple utterances. Narrative discourse in individuals with Parkinson's disease was analyzed by Reddy, Rao, and Narayanan (2016) which revealed that individuals with PD produced significantly less number of syntactic units compared to the control group. They also reported that individuals with PD had greater difficulty in producing complex language units such as sentences. The findings were in line with the findings of the current study.

\section{CONCLUSION}

The present study reveals that certain aspects of macrostructure and microstructure are affected in persons with PD. Thus, it can be concluded that in addition to speech impairment, language structure is also affected in persons with PD. It is important to focus on even the language aspects along with the motor speech aspects during the assessment and intervention in cases of neurodegenerative conditions. Intervening at the level of discourse is an important aspect as it enhances the quality of life. 
The limitation of this current research is that it includes smaller sample size. It is important to have the research extended with larger sample size to have a clear understanding about the macrostructural changes that could be observed in such individuals. It is also suggested that performance of the aspects comparing the post onset duration and conducting a longitudinal research on subjects as the disease progress would give us a clear picture of at what stage does the language symptoms emerge in individuals with Parkinson's disease.

\section{Acknowledgement: None}

\section{Conflict of Interest: None}

\section{Source of Funding: None}

\section{Ethical Approval: Approved}

\section{REFERENCES}

1. Amulya P. Rao, Madhusudarshan B. Reddy \& Swapna Narayanan (2017) Micro and macrostructure discourse analysis in persons with idiopathic Parkinson's disease, Speech, Language and Hearing, 20:4, 196-205, DOI: 10.1080/2050571X.2017.1303026

2. Angela Roberts; Danielle Post (2018). Information Content and Efficiency in the Spoken Discourse of Individuals With Parkinson's Disease. Journal of Speech,
Language, and Hearing Research, 61; https://doi.org/10.1044/2018_JSLHR-L-170338

3. Ash, S., Jester, C., York, C., Kofman, O. L., Langey, R., Halpin, A., ... \& Grossman, M. (2017). Longitudinal decline in speech production in Parkinson's disease spectrum disorders. Brain and language, 171, 42-51.

4. Madhu Sudharsan Reddy, Amulya P. Rao \& Swapna Narayanan (2016) Narrative discourse in persons with Parkinson's disease, Speech, Language and Hearing, 19:1, $1-9, \quad$ DOI: 10.1179/2050572815Y.0000000007

5. Murray, L. 2000. Spoken language production in Huntington's and Parkinson's diseases. Journal of Speech, Language, and Hearing Research 43: 1350-1366.

6. Roberts, Angela C., "Characterizing Spoken Discourse in Individuals with Parkinson Disease Without Dementia" (2014). Electronic Thesis and Dissertation Repository. 2336.

7. Zgaljardic, D. J., Foldi, N. S., \& Borod, J. C. (2004). Cognitive and behavioral dysfunction in Parkinson's disease: neurochemical and clinicopathological contributions. Journal of Neural Transmission, 111(10-11), 1287-1301.

How to cite this article: Maria J, Samyuktha N, Yuva Yoga Shree B et.al. Discourse analysis in Tamil speaking individuals with Parkinson's Disease. International Journal of Science \& Healthcare Research. 2021; 6(4): 11-16. DOI: https://doi.org/10.52403/ijshr.20211003 